J. Lake Sci. (湖泊科学), 2018, 30(1): 25-33

DOI 10. 18307/2018. 0103

(c) 2018 by Journal of Lake Sciences

\title{
蓝藻水华暴发期间太湖贡湖湾某水厂水源水及出厂水中微囊藻毒素 污染分析及健康风险评价
}

\author{
范亚民 ${ }^{1}$, 姜伟立 ${ }^{1}$, 刘宝贵 $^{2,3}$, 常闻捷 ${ }^{1}$, 吴召仕 ${ }^{2 * *}$ \\ ( 1 : 江苏省环境科学研究院江苏省环境工程重点实验室,南京 210036) \\ (2: 中国科学院南京地理与湖泊研究所湖泊与环境国家重点实验室,南京 210008) \\ (3: 中国科学院大学, 北京 100049)
}

\begin{abstract}
摘 要: 水体富营养化导致的有害蓝藻水华仍是目前全世界普遍面临的水环境问题, 而有害蓝藻水华所引起的饮用水安 全问题亦受到人们的广泛关注. 为了解太湖水源地水源水及自来水厂出厂饮用水中微囊藻毒素 $(\mathrm{MCs})$ 的污染现状, 于 2014 年 8 月期间对贡湖湾某水厂水源水及出厂水中浮游植物胞内及胞外 MCs 浓度进行了调查, 并同时检测了相关的理 化指标. 结果表明, 水源水中胞内 MCs 总浓度平均值为 $7165.5 \mathrm{ng} / \mathrm{L}$, 以 MC-LR 和 MC-RR 为主, 平均浓度分别为 3408.7 和 $3398.8 \mathrm{ng} / \mathrm{L}$, 其中 MC-RR 占总 MCs 比例的平均值为 $56.1 \%$; 而胞外溶解性 MCs 浓度相对较低, 平均浓度为 $142.6 \mathrm{ng} / \mathrm{L}$, 最 高浓度仅为 $512.8 \mathrm{ng} / \mathrm{L}$. 水厂出厂水中胞内 MCs 的检出浓度 (平均值为 $0.77 \mathrm{ng} / \mathrm{L}$ ) 和检出频率都很低, 去除率达 $99.8 \%$ 以 上; 而胞外溶解性 MCs 的检出浓度 (平均值为 $21.71 \mathrm{ng} / \mathrm{L}$ ) 和检出频率相对较高, 但浓度仍远低于国家标准 $1.0 \mu \mathrm{g} / \mathrm{L}$, 其去 除率相对较低, 仅为 $62.9 \% \sim 81.8 \%$. 数据分析发现, 水源水中胞内与胞外 MCs 浓度之间呈显著正相关, 胞内 MCs 浓度与 总氮 $(\mathrm{TN})$ 浓度、铵态氮 $\left(\mathrm{NH}_{4}^{+}-\mathrm{N}\right)$ 浓度、总磷 $(\mathrm{TP})$ 浓度、高锰酸盐指数 $\left(\mathrm{COD}_{\mathrm{Mn}}\right)$ 和浊度呈显著相关, 而胞外 $\mathrm{MCs}$ 浓度与 $\mathrm{TN}$ 浓度、TP 浓度、 $\mathrm{COD}_{\mathrm{Mn}}$ 、浊度和叶绿素 $\mathrm{a}$ 浓度呈显著正相关; 逐步回归结果显示, $\mathrm{TP}$ 对胞内 $\mathrm{MCs}$ 浓度变化的解释率最高, 而胞外 MCs 浓度变化主要与胞内 MCs 浓度相关. 最终, 通过对出厂饮用水中 MCs 浓度非致癌风险指数的计算发现, 出厂 饮用水对人类健康的威胁较小,但致癌风险相对较高.
\end{abstract}

关键词: 太湖; 贡湖湾; 蓝藻水华; 藻毒素; 潜在风险;饮用水安全;水源地

\section{Temporal microcystin dynamics of the source water and finished water in a waterworks of Lake Taihu}

\author{
FAN Yamin ${ }^{1}$, JIANG Weili ${ }^{1}$, LIU Baogui ${ }^{2,3}$, CHANG Wenjie ${ }^{1} \&$ WU Zhaoshi ${ }^{2 * *}$ \\ (1: Jiangsu Province Key Laboratory of Environmental Engineering, Jiangsu Provincial Academy of Environmental Science, \\ Nanjing 210036, P.R.China) \\ (2: State Key Laboratory of Lake Science and Environment, Nanjing Institute of Geography and Limnology, Chinese Academy \\ of Sciences, Nanjing 210008, P.R.China) \\ (3: Graduate University of Chinese Academy of Sciences, Beijing 100049, P.R.China)
}

\begin{abstract}
Harmful cyanobacteria blooms, which are caused by water eutrophication, is still one of the serious water environment problems encountered by the whole world. In order to estimate the current status of microcystins (MCs) in the source water and finished water of Lake Taihu, we examined the concentrations of intracellular MC (intraMCs) and extracellular MC (extraMCs) and related physiochemical parameters of the source water and finished water in a waterworks in August 2014. Our results showed that the mean concentration of intraMCs in the source water was $7165.5 \mathrm{ng} / \mathrm{L}$, dominated by MC-LR and MC-RR, with the mean concentration of 3408.7 and $3398.8 \mathrm{ng} / \mathrm{L}$, respectively, and MC-RR accounted for $56.1 \%$ of the total MCs. The extraMCs in the source water were still on a low level with the mean and maximum value of 142.6 and $512.8 \mathrm{ng} / \mathrm{L}$, respectively. However, the con-
\end{abstract}

* 国家水体污染控制与治理科技重大专项 (2012ZX07506-008-01,2014ZX07101-011) 资助. 2017-03-19 收稿; 2017 06-06 收修改稿. 范亚民 (1977 ), 男, 博士研究生; E-mail : fanyamin@163.net.

** 通信作者; E-mail: zswu@ niglas.ac.cn. 
centrations of intraMCs in finished water were relatively on a low level (mean value of $0.77 \mathrm{ng} / \mathrm{L}$ ) with the removal efficiency above $99.98 \%$. Although extraMCs in finished water were also detected on a low level (mean value of $21.71 \mathrm{ng} / \mathrm{L}$ ), the concentration and detection frequencies of extraMCs were significantly higher than those of intraMCs with removal efficiency of $62.9 \%-$ $81.8 \%$. Data analysis showed that the significant and positive correlation was observed between concentrations of intraMCs and extraMCs in source water. Meanwhile, intraMCs were significantly correlated with total nitrogen $(\mathrm{TN})$, ammonium $\left(\mathrm{NH}_{4}^{+}-\mathrm{N}\right)$, total phosphorus $(\mathrm{TP})$, chemical oxygen demand $\left(\mathrm{COD}_{\mathrm{Mn}}\right)$ and turbidity, while extraMCs were significantly and positively correlated with TN, TP, $\mathrm{COD}_{\mathrm{Mn}}$, turbidity and chlorophyll-a. In addition, according to stepwise multiple linear regression analysis, TP could explain most of the variance of intraMCs in source water, while the variations of extraMCs could be primarily explained by intraMCs. Finally, according to non-carcinogenic risk index, MCs in finished water presented a relative low threat to the people there, while the risks will be higher when considering carcinogenic risk.

Keywords: Lake Taihu; Gonghu Bay; cyanobacteria blooms; microcystins; potential risk; drinking water safety; water source area

目前, 由水体富营养化导致的淡水水体有害蓝藻水华仍是全世界普遍面临的水环境问题 ${ }^{[1]}$. 近几十年 来, 我国的富营养化问题日益严重,在对 138 个面积大于 $10 \mathrm{~km}^{2}$ 的湖泊进行调查之后发现,其中 $85.4 \%$ 的湖 泊已处于富营养化状态, 而其中更高达 $40.1 \%$ 的湖泊已处于重度富营养化状态 ${ }^{[2]}$. 湖泊富营养化会产生一 系列环境问题, 而蓝藻水华便是其中研究最多且污染最为严重的一种. 蓝藻水华的暴发不仅会对水质产生 显著影响, 还会产生一系列毒性很强的次级代谢产物, 严重危害人类健康. 其中, 以微囊藻毒素的危害最为 严重 ${ }^{[3]}$.

微囊藻毒素 $(M C s)$ 是世界各地水华蓝藻产生的最为常见且危害最严重的蓝藻毒素 ${ }^{[4-5]}$, 目前已确认的 产毒蓝藻主要有微囊藻属、浮丝藻属、颤藻属、念珠藻属和鱼腥藻属等 ${ }^{[5]}$. 研究表明, MCs 为环状七肽结构, 自 1980s 初确定结构以来,已发现 90 余种毒素亚型, 其中以 MC-LR、MC-RR 和 MC-YR 的研究最多, 而以 MC-LR 的毒性最强 ${ }^{[6-8]}$. 大量文献报道指出, MCs 具有很强的肝脏毒性, 同时, 大多数的 MCs 具有亲水性, 在 水中的溶解度最高可达 $1 \mathrm{~g} / \mathrm{L}^{[9]}$. 此外, $\mathrm{MCs}$ 还具有很高的热稳定性, 在 $300^{\circ} \mathrm{C}$ 条件下仍能维持很长时间不分 解 ${ }^{[10]}$. 目前, 已有许多关于动物以及人类 MCs 中毒的报道, 其中最为严重的事件为 1996 年巴西 Carurau 透 析中心的 MCs 中毒事件, 最终确认有 52 人的死亡与 MCs 中毒有关 ${ }^{[11-12]}$. 此外, 流行病学的研究发现, 我国 南方某些地区 (如江苏泰兴 ${ }^{[13]}$ 和海门 ${ }^{[14]}$ 等) 原发性肝癌的高发病率与饮用水被 MCs 污染相关.

太湖作为中国第三大淡水湖,因水体富营养化而导致的蓝藻水华问题由来已久,在 2007 年左右发生蓝 藻水华的湖面面积达到最大值 ( $1000 \mathrm{~km}^{2}$ 以上), 并导致 2007 年无锡饮用水危机事件的发生 ${ }^{[1,15]}$. 虽然最近 几年太湖蓝藻水华暴发的湖面面积没有进一步扩大, 但由于太湖的季风气候等特点, 在太湖北部富营养化 严重区域仍频繁发生较大面积的蓝藻聚集 ${ }^{[16]}$. 而 MCs 作为蓝藻的重要有毒代谢产物, 对太湖已造成较为严 重的污染, 已有研究表明, 太湖中 MC-LR 的平均浓度已达到 $1.48 \mu \mathrm{g} / \mathrm{L}$, 最高达 $2.558 \mu \mathrm{g} / \mathrm{L}$, 这已显著高出世 界卫生组织和我国规定的饮用水含量标准 ( GB 5749-2006,1.0 $\mu \mathrm{g} / \mathrm{L}$ ). 目前, 对太湖水体中 MCs 的研究已 较多, 但多集中于对太湖全湖以及蓝藻水华暴发区域的研究 ${ }^{[17-18]}$, 对太湖饮用水源地和水厂出厂水中的 MCs 分布特征及影响因素关注较少. 此外, 以往研究多为全年的调查, 频度较低, 蓝藻暴发阶段 MCs 的变化 规律仍不清楚, 并集中于对单一 MCs 异构体 MC-LR 的研究 ${ }^{[19]}$, 而 MC-RR 浓度在太湖水中亦占相当大的比 例. 有鉴于此, 我们对太湖北部贡湖湾区域某水厂水源地水源水和出厂饮用水胞内 $\mathrm{MCs}$ 以及胞外 MCs 浓度 进行了高频检测, 在分析 MCs 浓度随时间变化规律的基础上, 对影响其浓度变化的环境因子进行分析, 并对 其进行相应的健康风险评价.

\section{1 材料与方法}

\section{1 采样点和采样时间}

本研究采样点位于太湖北部贡湖湾湾口, 沙渚水源地, 而水厂地处江苏省无锡市范围内. 在 2014 年 8 月蓝藻水华暴发期间, 对目标水厂进行了为期一个月的调查, 采样点设定在水厂取水口和出水口处. 从 8 月 1 日开始每隔 1 天采集 1 次样品,到 8 月 29 日截止,共采集样品 15 次. 


\section{2 样品采集及预处理}

在取水口附近湖面, 采用 Hydrolab (美国) 公司生产的 DS5X 型水质多功能探头现场测定水温、 $\mathrm{pH}$ 、溶解 氧 (DO)、浊度、矿化度、盐度等指标. 同时, 用 $2.5 \mathrm{~L}$ 有机玻璃采水器采集混合水样 $1 \mathrm{~L}$ 用于分析胞内和胞外 $\mathrm{MCs}$ 浓度. 此外, 采集 $1 \mathrm{~L}$ 混合水用于检测总氮 $(\mathrm{TN})$ 、铵态氮 $\left(\mathrm{NH}_{4}^{+}-\mathrm{N}\right)$ 、总磷 $(\mathrm{TP})$ 、高锰酸盐指数 $\left(\mathrm{COD}_{\mathrm{Mn}}\right)$ 和叶绿素 a(Chl.a) 浓度.

将采集的部分水样用 Whatman $\mathrm{GF} / \mathrm{C}(0.45 \mu \mathrm{m})$ 滤膜过滤, 滤后水样用于检测胞外 $\mathrm{MCs}$ 和 $\mathrm{NH}_{4}^{+}-\mathrm{N}$ 浓度, 滤膜用于检测胞内 MCs 和 Chl.a 浓度.

\section{3 参数测定}

胞内和胞外 MCs 浓度的检测参考文献 $[8,18]$. 首先, 用 $5 \%$ 的乙酸将冷冻干燥后的滤膜超声萃取 5 $\mathrm{min}$, 在悬浮液冷冻离心之后, 将上清液加到 $\operatorname{HLB}(200 \mathrm{mg}$, Oasis (C), Waters, Milford, MA, USA) 萃取柱内. 在萃取柱内液面将要消失时, 分别用 $20 \mathrm{ml} 5 \%$ 的甲醇和 $12 \mathrm{ml} \mathrm{100 \%}$ 的甲醇清洗和洗脱柱子. 然后, 将洗脱液 在 $40^{\circ} \mathrm{C}$ 条件下用氮气吹干. 最后, 用 $1 \mathrm{ml} 100 \%$ 的甲醇将残留物溶解, 取其中的 $0.5 \mathrm{ml}$ 检测胞内 $\mathrm{MCs}$ 浓度. 将用于检测胞外 MCs 浓度的滤液直接添加到 HLB 柱子内, 而后面的步骤与以上胞内 MCs 检测步骤相同. 在 以上的萃取浓缩步骤结束之后, 使用高效液相色谱仪 (HPLC, Agilent 1200 series, Palo Alto, CA, USA) 分析 其中的 $\mathrm{MCs}\left(-\mathrm{LR} 、-\mathrm{RR}\right.$ 和-YR) 浓度. 高效液相色谱仪配备 $\mathrm{C}_{18}(\mathrm{ODS})$ 反相色谱柱和光电二极管阵列检测器, 梯度洗脱程序为 $30 \% \sim 40 \%$ 乙腈和 $60 \% \sim 70 \%$ 的 $0.05 \%$ 三氟乙酸 ( $15 \mathrm{~min}$ ), 之后乙腈与三氟乙酸溶液按体积 比 $30: 70$ 混合 (5 min), 流速为 $1 \mathrm{ml} / \mathrm{min}$. 仪器检测时使用的标准品购买于 Sigma-Aldrich (München, Germany).

$\mathrm{TN}$ 和 TP 浓度采用过硫酸钾消解法测定 ${ }^{[20]}, \mathrm{NH}_{4}^{+}-\mathrm{N}$ 浓度应用荷兰 Skalar 公司生产的连续流动分析仪测 定,Chl.a 浓度通过热乙醇分光光度法测定 ${ }^{[21]}$.

\section{4 健康风险评价方法}

本研究对出厂水中胞外 MCs 的健康风险评价采用风险指数 $(\mathrm{HI})$ 法进行计算, 具体计算方法参照王超 等 ${ }^{[22]}$ 和尤汉虎等 ${ }^{[23]}$ 文献中的描述.

\section{5 数据分析}

使用 SPSS 20.0 软件对数据进行标准化、Spearman 相关分析和逐步回归分析. 其中, 在进行逐步回归分 析时,为避免自变量的多重共线性,去掉相关分析中任意两个自变量相关系数大于 0.8 的其中一个自变量. 绘图软件使用 Origin 9.0 软件.

\section{2 结果与分析}

\section{1 水源水胞内和胞外 MCs 浓度的变化}

水源水中胞内 MCs 的 3 种异构体与总 MCs 浓度变化具有较高的一致性, 均具有相似的波动规律, 且均 在下旬时浓度较高, 3 种 MCs 异构体以 MC-LR 和 MC-RR 为主, 分别占总 MCs 的 38.7\% 和 56.1\%. 调查期间, MC-LR 的平均浓度为 $3408.7 \mathrm{ng} / \mathrm{L}$, 浓度范围为 $107 \sim 15379 \mathrm{ng} / \mathrm{L}$, 最大值出现在 8 月 21 日; 在前 10 次调查 中, MC-LR 浓度明显低于 MC-RR, 而在调查后期其浓度略有升高. MC-RR 在调查期间的平均浓度为 3398.8 $\mathrm{ng} / \mathrm{L}$, 浓度范围为 $364.1 \sim 6470.4 \mathrm{ng} / \mathrm{L}$, 其最大值出现在 8 月 25 日. 在调查期间 MC-YR 浓度较低, 平均值为 $358 \mathrm{ng} / \mathrm{L}$, 浓度范围为 $21.7 \sim 1356.7 \mathrm{ng} / \mathrm{L}$ (图 1a).

在调查期间, 水源水胞外 MCs 浓度显著低于胞内 MCs 浓度, 并与胞内 MCs 具有相似的变化规律, 其平 均浓度为 $142.6 \mathrm{ng} / \mathrm{L}$, 浓度范围为 11.9 512.8 ng/L. 胞外 MCs 3 种异构体浓度的变化规律相同, 均在 8 月 23 日时达到最大值, 相对胞内 MCs 最大值出现的日期略晚, MC-LR、MC-RR 和 MC-YR 所占比例分别为 $50.9 \%$ 、 $36.1 \%$ 和 $13.1 \%$. 胞外 MC-LR 的平均浓度为 $65.7 \mathrm{ng} / \mathrm{L}$, 浓度范围为 $6.4 \sim 216.5 \mathrm{ng} / \mathrm{L} ; \mathrm{MC}-\mathrm{RR}$ 的平均浓度为 $63.5 \mathrm{ng} / \mathrm{L}$, 浓度范围为 $1.7 \sim 262.1 \mathrm{ng} / \mathrm{L}$; 而 $\mathrm{MC}-\mathrm{YR}$ 的平均浓度仅为 $13.5 \mathrm{ng} / \mathrm{L}$, 浓度范围为 $3.2 \sim 34.2 \mathrm{ng} / \mathrm{L}$ (图 1b).

\section{2 出厂饮用水胞内和胞外 MCs 浓度的变化及去除率分析}

在调查期间, 出厂饮用水中胞内 MCs 仅检测到 MC-LR 与 MC-RR 两种毒素异构体, 且毒素浓度都非常 

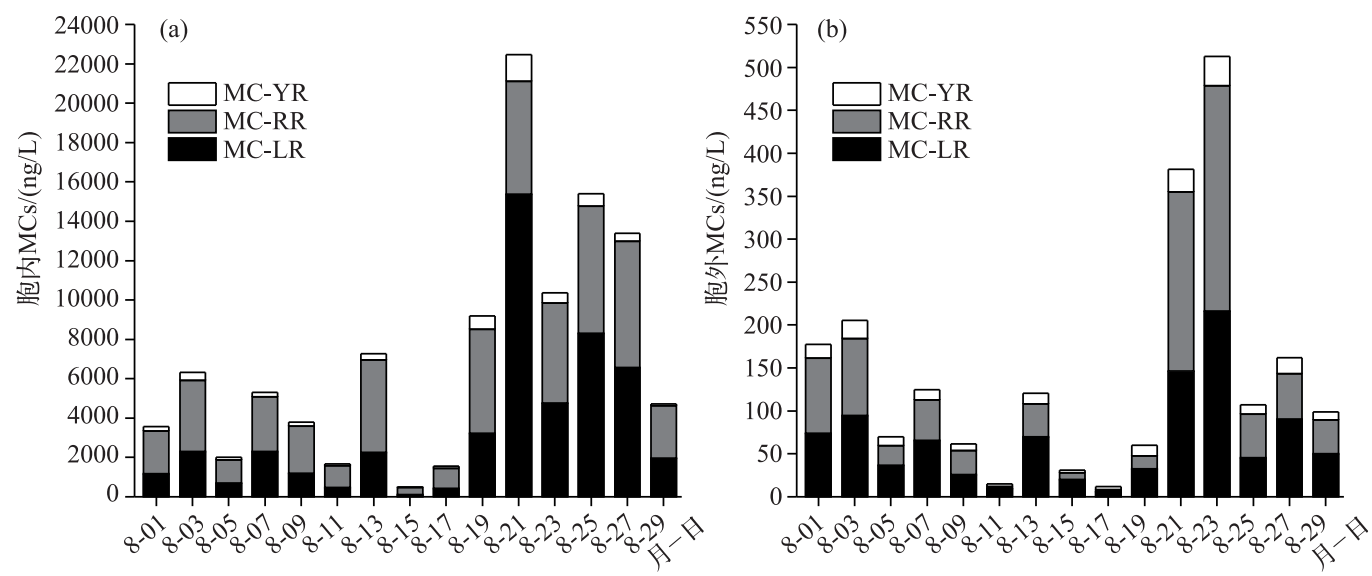

图 1 水源水胞内及胞外 MCs 浓度的变化

Fig. 1 Temporal variation of intraMCs and extraMCs concentrations of the source water

低, 远低于国家饮用水标准规定的 $1 \mu \mathrm{g} / \mathrm{L}$. 其中, 仅有 3 次检测到 MC-LR, 平均浓度为 $0.25 \mathrm{ng} / \mathrm{L}$, 浓度范围 为 $0 \sim 1.64 \mathrm{ng} / \mathrm{L}$, 最大值出现在 8 月 25 日; 而 MC-RR 的检出次数明显高于 MC-LR, 平均浓度为 $0.53 \mathrm{ng} / \mathrm{L}$, 浓 度范围为 $0 \sim 3.19 \mathrm{ng} / \mathrm{L}$, 最大值出现在 8 月 23 日 (图 2a). 相对于胞内 MCs, 胞外 MCs 的浓度与检出频率均 显著高于胞内 MCs, 但浓度最大值均出现在 8 月 3 日, 除 MC-YR 之外, 其他两种 MCs 异构体和总 MCs 浓度 均在 8 月 25 日出现另一浓度高值. 其中, MC-LR 的平均浓度为 $9.47 \mathrm{ng} / \mathrm{L}$, 浓度范围为 $0 \sim 30.56 \mathrm{ng} / \mathrm{L}$; MC-RR 的平均浓度为 $10.46 \mathrm{ng} / \mathrm{L}$, 浓度范围为 $0 \sim 46.36 \mathrm{ng} / \mathrm{L}$; 而 MC-YR 的浓度相对更低, 平均浓度为 $1.78 \mathrm{ng} / \mathrm{L}$, 浓 度范围为 $0 \sim 5.91 \mathrm{ng} / \mathrm{L}$ (图 $2 \mathrm{~b}$ ). 对数据的分析可以发现, 饮用水中胞外 MCs 浓度更高, 且在调查期间都有 $\mathrm{MCs}$ 检出, 总 MCs 的平均浓度为 $21.71 \mathrm{ng} / \mathrm{L}$, 浓度范围为 $0.18 \sim 82.84 \mathrm{ng} / \mathrm{L}$.
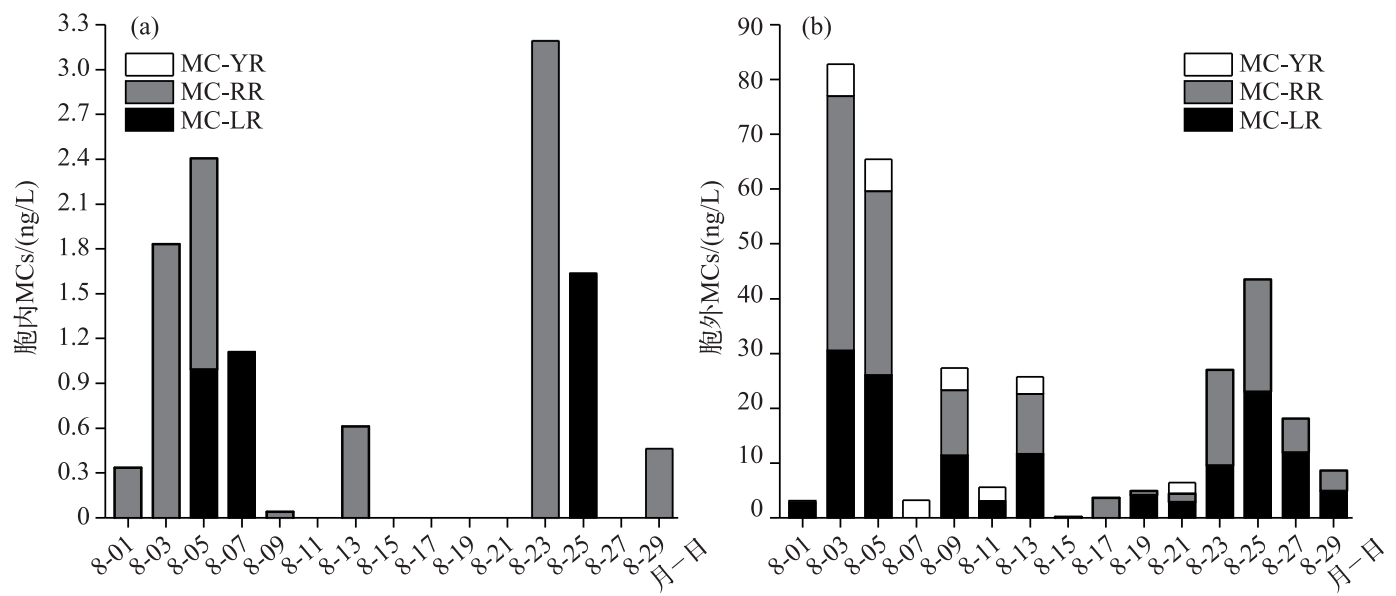

图 2 出厂饮用水胞内和胞外 MCs 浓度的变化

Fig.2 Temporal variation of intraMCs and extraMCs concentrations of the finished water

进一步对 MCs 的去除率( 图 3) 进行分析发现, 水厂对胞内 MCs 的去除率非常高, 3 种 MCs 异构体以及 总 MCs 的去除率平均值均达到 $99.8 \%$ 以上; 而对胞外 MCs 的去除率相对较低, MC-LR、MC-RR、MC-YR 及总 MCs 的平均去除率分别为 $80.6 \% 、 62.9 \% 、 81.8 \%$ 和 76.8\% . 此外, 在其中几次调查中胞外 MCs 的去除率较低 或者为负值,这说明水厂对水源水的处理过程可能导致了胞内 MCs 的释放. 


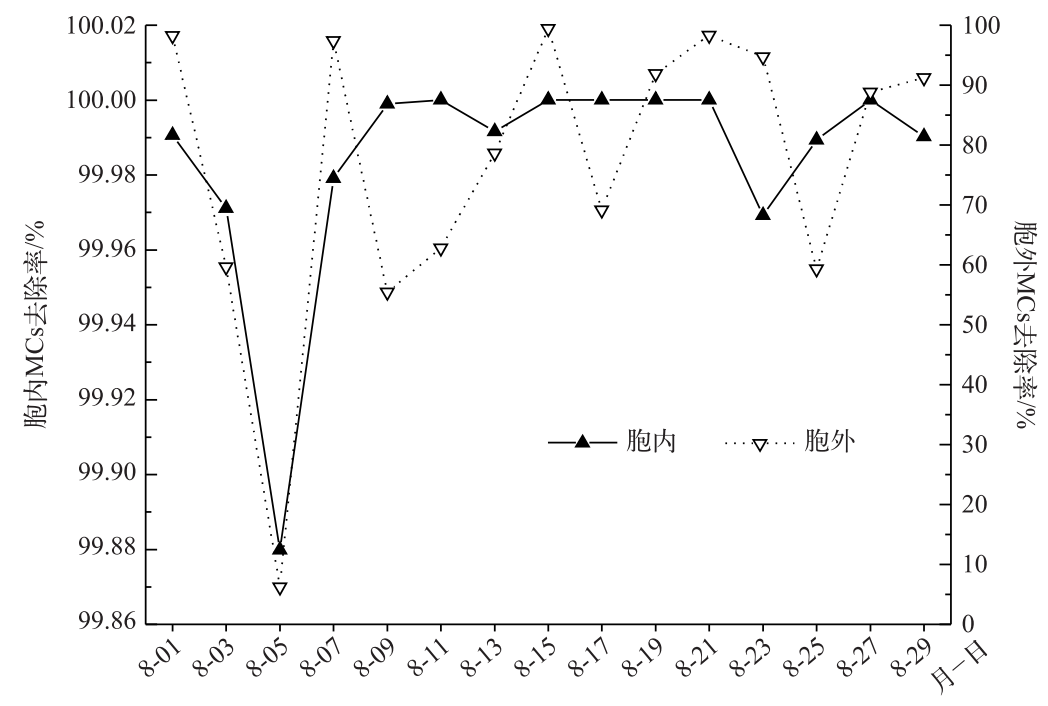

图 3 自来水厂对水源地水中胞内和胞外 MCs 的去除率

Fig.3 Removal rates of total intraMCs and extraMCs by the waterworks

\section{3 水源水 MCs 浓度变化的影响因子分析}

调查期间水源水的主要理化参数如表 1 所示. 同时, 对水源水胞内与胞外 MCs 浓度和其他理化指标进 行 Spearman 相关分析 (表 2) 发现,胞内不同 MCs 异构体与胞外不同 MCs 异构体之间具有显著相关性. 此 外, 胞内 MCs 浓度与 TN 浓度、TP 浓度、 $\mathrm{COD}_{\mathrm{Mn}}$ 浓度和浊度呈显著正相关, 其中, MC-YR 浓度与 $\mathrm{NH}_{4}^{+}-\mathrm{N}$ 浓度

表 1 调查期间水源水理化参数

Tab.1 Physiochemical parameters of the source water during the survey

\begin{tabular}{|c|c|c|c|c|c|c|c|c|c|c|}
\hline 参数 & $\begin{array}{c}\mathrm{TN} / \\
(\mathrm{mg} / \mathrm{L})\end{array}$ & $\begin{array}{l}\mathrm{NH}_{4}^{+}-\mathrm{N} / \\
(\mathrm{mg} / \mathrm{L})\end{array}$ & $\begin{array}{c}\mathrm{TP} / \\
(\mathrm{mg} / \mathrm{L})\end{array}$ & $\begin{array}{l}\mathrm{COD}_{\mathrm{Mn}} \text { ' } \\
(\mathrm{mg} / \mathrm{L})\end{array}$ & 水温 $/{ }^{\circ} \mathrm{C}$ & $\begin{array}{c}\mathrm{DO} / \\
(\mathrm{mg} / \mathrm{L})\end{array}$ & $\begin{array}{l}\text { 电导率/ } \\
\text { (mS/cm) }\end{array}$ & $\begin{array}{c}\text { 浊度/ } \\
\text { NTU }\end{array}$ & $\mathrm{pH}$ & $\begin{array}{l}\text { Chl.a/ } \\
(\mu \mathrm{g} / \mathrm{L})\end{array}$ \\
\hline 平均值 & $3.89 \pm 5.87$ & $0.25 \pm 0.18$ & $0.32 \pm 0.54$ & $11.38 \pm 16.56$ & $27.72 \pm 2.30$ & $5.76 \pm 1.70$ & $0.52 \pm 0.01$ & $157.9 \pm 325.0$ & $8.16 \pm 0.32$ & $137.6 \pm 225.8$ \\
\hline
\end{tabular}

表 2 水源水 MCs 浓度与其他参数的相关性分析 ${ }^{1)}$

Tab.2 Spearman's correlation analysis between MCs concentrations and other parameters of the source water

\begin{tabular}{ccccccccc}
\hline 相关性 & SILR & SIRR & SIYR & SITMC & SELR & SERR & SEYR & SETMC \\
\hline SELR & $0.714^{* *}$ & $0.639^{*}$ & $0.646^{* *}$ & $0.707^{* *}$ & - & $0.950^{* *}$ & $0.921^{* *}$ & $0.986^{* *}$ \\
SERR & $0.736^{* *}$ & $0.643^{* *}$ & $0.657^{* *}$ & $0.707^{* *}$ & $0.950^{* *}$ & - & $0.871^{* *}$ & $0.975^{* *}$ \\
SEYR & $0.782^{* *}$ & $0.721^{* *}$ & $0.825^{* *}$ & $0.771^{* *}$ & $0.921^{* *}$ & $0.871^{* *}$ & - & $0.900^{* *}$ \\
SETMC & $0.696^{* *}$ & $0.607^{*}$ & $0.636^{*}$ & $0.679^{* *}$ & $0.986^{* *}$ & $0.975^{* *}$ & $0.900^{* *}$ & - \\
TN & $0.675^{* *}$ & $0.596^{*}$ & $0.536^{*}$ & $0.646^{* *}$ & $0.600^{*}$ & $0.654^{* *}$ & 0.496 & $0.657^{* *}$ \\
$\mathrm{NH}_{4}^{+}-\mathrm{N}$ & $-0.422^{* *}$ & $-0.338^{* *}$ & $-0.522^{*}$ & -0.386 & -0.315 & -0.449 & -0.422 & -0.347 \\
$\mathrm{TP}$ & $0.707^{* *}$ & $0.632^{*}$ & $0.604^{*}$ & $0.675^{* *}$ & $0.621^{*}$ & $0.661^{* *}$ & $0.564^{*}$ & $0.675^{* *}$ \\
$\mathrm{COD}$ & $0.657^{* *}$ & $0.575^{*}$ & $0.571^{*}$ & $0.629^{*}$ & $0.579^{*}$ & $0.629^{*}$ & $0.521^{*}$ & $0.643^{* *}$ \\
浊度 & $0.700^{* *}$ & $0.607^{*}$ & $0.600^{*}$ & $0.675^{* *}$ & $0.793^{* *}$ & $0.786^{* *}$ & $0.704^{* *}$ & $0.825^{* *}$ \\
$\mathrm{Chl} . \mathrm{a}$ & 0.306 & 0.220 & 0.382 & 0.263 & $0.634^{*}$ & $0.652^{* *}$ & $0.627^{*}$ & $0.710^{* *}$ \\
\hline
\end{tabular}

1) SI ( LR、RR、YR 和 TMC) : 分别代表水源水胞内 MCs 的 3 种异构体和总 MCs 浓度; SE ( LR、RR、YR 和 TMC) : 分别代表 水源水胞外 MCs 3 种异构体和总 MCs 浓度; $*$ 代表 $P<0.05$, ** 代表 $P<0.01$;下同. 
呈显著负相关, 而不同 MCs 异构体浓度与 Chl.a 浓度的相关性均不显著; 胞外 MCs 浓度与 TN 浓度、TP 浓 度、 $\mathrm{COD}_{\mathrm{Mn}}$ 浓度、浊度和 Chl.a 浓度呈显著正相关, 与 $\mathrm{NH}_{4}^{+}-\mathrm{N}$ 浓度同样呈负相关, 但不显著.

水源水中胞内和胞外 MCs 与其他理化指标的逐步回归分析结果如表 3 所示, 其中, 胞内 MC-RR 做因变 量时无自变量被选出. 从表中可以看出, 胞内 MC-LR 和总 MCs 做因变量时, 仅有 TP 对其有显著的影响, 解 释率分别为 74.7\% 和 56.4\% ; TP 和胞外 MC-YR 对胞内 MC-YR 浓度的影响最为显著, 且为正相关, 解释率分 别为 $69.4 \%$ 和 $9.3 \%$. 胞外 MC-LR 做因变量时, 仅有胞内 MC-LR 被篮出, 解释率为 $31.4 \%$; 而胞外 MC-RR 做因 变量时, 有 4 个变量被笁出, 其中 TP、胞内 MC-RR 和出厂水胞内 MC-RR 与其呈正相关, 而出厂水胞外 MC-RR 与其呈负相关, 解释率依次为 $42.4 \% 、 4.1 \% 、 37.9 \%$ 和 $8.1 \%$; 胞外 MC-YR 做因变量时, 仅有胞内 MC-YR 与其 呈显著相关, 解释率为 $42.2 \%$; 当胞外总 MCs 做因变量时, 胞内总 MCs 以及出厂水胞内总 MCs 被选出, 且都 为正相关关系,解释率分别为 $37.9 \%$ 和 $22.4 \%$.

表 3 水源水胞内和胞外 MCs 与其他理化指标的逐步回归分析结果 *

Tab.3 Results of stepwise multiple linear regression between intraMCs and extraMCs concentrations and other physiochemical parameters of the source water

\begin{tabular}{|c|c|c|c|c|c|c|c|}
\hline & SILR & SIYR & SITMC & SELR & SERR & SEYR & SETMC \\
\hline $\mathrm{TP}$ & 0.864 & 0.678 & 0.751 & - & 0.552 & - & - \\
\hline SILR & - & - & - & 0.560 & - & - & - \\
\hline SIRR & - & - & - & - & 0.223 & - & - \\
\hline SIYR & - & - & - & - & - & 0.649 & - \\
\hline SITMC & - & - & - & - & - & - & 0.587 \\
\hline SEYR & - & 0.341 & - & - & - & - & - \\
\hline ZIRR & - & - & - & - & 0.920 & - & - \\
\hline ZITMC & - & - & - & - & - & - & 0.474 \\
\hline ZERR & - & - & - & - & -0.390 & - & - \\
\hline Adjusted $R^{2}$ & 0.727 & 0.751 & 0.531 & 0.261 & 0.896 & 0.377 & 0.536 \\
\hline$P$ & $<0.01$ & $<0.01$ & $<0.01$ & 0.03 & $<0.01$ & $<0.01$ & $<0.01$ \\
\hline$V I F$ & $<3$ & $<3$ & $<3$ & $<3$ & $<3$ & $<3$ & $<3$ \\
\hline
\end{tabular}

* ZI ( LR、RR、YR 和 TMC) : 分别代表出厂水胞内 MCs 的 3 种异构体和总 MCs 浓度; ZE ( LR、RR、YR 和 TMC) : 分别代表 出厂水胞外 MCs 的 3 种异构体和总 MCs 浓度; Adjusted $R^{2}$ : 回归模型的校正决定系数; $P<0.05$ 时代表回归模型有效; VIF (variation inflation factor) : 方差膨胀因子,当 $V I F<10$ 时,表明各自变量之间多重共线性对模型不存在影响.

\section{4 水厂出厂饮用水中 MCs 浓度的健康风险评价}

经过计算, 调查期间, 目标水厂出厂饮用水 MCs 的非致癌风险指数变化范围为 $5.67 \times 10^{-6} \sim 2.761 \times 10^{-3}$, 均远小于 1 , 所以, 出厂水中 MCs 浓度对人类健康所造成的风险较小.

\section{3 讨论}

MCs 主要为蓝藻门中特定产毒藻产生的胞内毒素, 仅在蓝藻细胞衰老等条件导致细胞破裂时才会释放 到周围的环境中 ${ }^{[24]}$, 所以当水体中具有较高浓度的胞内 MCs 时, 胞外溶解性 MCs 也会相应较高, 但浓度最 大值出现的时间相对胞内 MCs 浓度最大值出现的时间要略晚, 这与本研究结果一致. 调查结果发现, 目标水 厂水源水中胞内 MCs 总浓度已经处在非常高的水平, 浓度最高已达 $22461.3 \mathrm{ng} / \mathrm{L}$, 且平均浓度也处在较高 水平. $\mathrm{Su}$ 等 ${ }^{[18]}$ 在 2013 年 7 月-2014 年 6 月期间对太湖北部区域的调查发现,胞内 MCs 在 7 月达到最高值, 平均浓度达到 $10930 \mathrm{ng} / \mathrm{L}$, 而在 8 月浓度 $(<4 \mu \mathrm{g} / \mathrm{L})$ 显著下降, 在 10 月又升到另一峰值. 然而, 本研究发现, 水源水中胞外毒素浓度在调查期间一直处于较低水平, 显著低于国家饮用水标准规定的 $1 \mu \mathrm{g} / \mathrm{L}$, 这与唐承 佳 ${ }^{[25]}$ 对太湖贡湖湾的研究结果一致. 而高振美等 ${ }^{[26]}$ 对太湖梅梁湾的调查结果则显著高出本研究结果, 在湖 区的某些区域 MCs 的总浓度甚至超过了 $1 \mu \mathrm{g} / \mathrm{L}$, 王经结等 ${ }^{[17]}$ 对太湖的研究亦得到相似的结果. 虽然以上研 究结果中总 MCs 浓度已超过 $1 \mu \mathrm{g} / \mathrm{L}$, 但是 MC-LR 浓度仍处于较低水平, 而在毛敬英 ${ }^{[27]}$ 对太湖的研究中, 胞 
外 MC-LR 的平均浓度已达到 $1.48 \mu \mathrm{g} / \mathrm{L}$, 最高浓度甚至达到 $2.558 \mu \mathrm{g} / \mathrm{L}$. 所以, 综上所述, 太湖北部区域水源 地水源水中 MCs 普遍存在, 尤其在蓝藻暴发季节, 某些区域的 MCs 浓度已经接近或超过国家饮用水标准规 定的限值. 有鉴于此,相关部门应当加强对太湖相关区域的常规监测, 并对 MCs 可能造成的风险进行相关评 价和预警.

然而, 目前对 MCs 的检测还大多集中于水源地及其所在湖区,而对自来水厂出厂饮用水的检测相对较 少. 本研究调查发现, 目标水厂对水源水中胞内 MCs 具有很高的去除率, 经处理后的自来水中仅含有微量的 胞内 MCs, 而胞外 MCs 去除率相对较低, 浓度亦较高. 相关研究表明 ${ }^{[28-29]}$, 传统的混凝、沉淀、过滤和加氯等 净水工艺过程只能去除部分胞内 MCs, 对胞外溶解性 MCs 的去除作用不大, 有时一些处理过程还会因为导 致蓝藻细胞破裂而使胞外 MCs 浓度升高, 这也可能是本研究中胞外 MCs 去除率相对较低的原因之一. 虽然 出厂饮用水中 MCs 浓度较低, 但是胞外 MCs 的检出频率非常高, 不排除在长时间暴露条件下对附近居民存 在长期的毒性作用, 所以今后应在加强自来水处理的基础上, 进一步加强对 MCs 长期暴露毒性实验的研究. 此外, 由于目前使用的《地表水环境质量标准》和《生活饮用水卫生标准》中仅规定了 MC-LR 的限值, 而对其 他 MCs 异构体浓度限值却没有涉及, 所以今后还应加强对其他主要 MCs 异构体的研究, 进一步完善饮用水 等的评价标准.

目前, 对 MCs 时空分布影响因子的研究已经有很多, 但是由于研究条件不统一, 导致研究结果也各不相 同. 水体中氮、磷等营养物质浓度对其中产毒藻类的生长具有重要影响, 研究发现, 当 TP 浓度在一定范围内 时, 产毒微囊藻生物量随 TP 浓度的升高而升高 ${ }^{[30]}$, 所以, 水体中 MCs 的浓度也会随之升高. 本研究逐步回 归分析结果显示, 理化指标仅有 TP 对胞内 MCs 浓度的影响最为显著, 与胞内 MCs 浓度呈显著正相关, 这与 Rinta-Kanto 等 ${ }^{[31]}$ 以及 Lee 等 ${ }^{[32]}$ 的研究一致, 而亦有研究表明磷浓度与 MCs 浓度不存在显著相关关系 ${ }^{[33-34]}$. 对于其他理化因子, 相关的研究也有很多, 但主要集中于 $\mathrm{TN}$ 、光照强度、水温、藻类生物量 (Chl.a) 以及细胞 生长速率等. 而根据本研究结果, 今后应进一步加强对磷与 MCs 浓度之间关系的研究, 同时, 加强对新技术 的研发, 能够在去除胞内 MCs 的基础上,减少胞外 MCs 的释放.

最后, 通过对水厂出厂饮用水中 MCs 浓度非致癌风险指数的计算, 表明出厂水中 MCs 对人类健康存在 的危害仍较小. 然而, 相关研究指出, MCs 亦具有一定的促癌作用 ${ }^{[35-36]}$, 这使得由 MCs 引起的健康风险指数 显著升高,但目前仍缺少对 MCs 致癌斜率因子的研究,致使 MCs 的致癌风险指数不能量化.

\section{4 参考文献}

[ 1 ] Qin BQ, Yang GJ, Ma JR et al. Dynamics of variability and mechanism of harmful cyanobacteria bloom in Lake Taihu, China. Chin Sci Bull, 2016, 61(7) : 759-770. [秦伯强, 杨桂军, 马健荣等. 太湖蓝藻水华 “暴发” 的动态特征及其 机制. 科学通报, 2016, 61(7): 759-770.]

[ 2 ] Yang GS, Ma RH, Zhang L et al. Lake status, major problems and protection strategy in China. J Lake Sci, 2010,22 (6), 799-810. DOI: 10.18307/2010.0601. [杨桂山, 马荣华, 张路等. 中国湖泊现状及面临的重大问题与保护策 略. 湖泊科学, 2010, 22(6): 799-810.]

[ 3 ] Xie P. A review on the studies related to the effects of microcystins on human health. J Lake Sci, 2009, 21( 5) : 603-613. DOI: 10.18307/2009.0501. [谢平. 微囊藻毒素对人类健康影响相关研究的回顾. 湖泊科学, 2009, 21 (5): 603-613.]

[ 4 ] Xue QJ, Su XM, Xie LQ. Advances on cyanotoxin toxicology of zoobenthos. Acta Ecol Sin, 2015, 35(14): 4570-4578. [薛庆举, 苏小妹, 谢丽强. 蓝藻毒素对底栖动物的毒理学研究进展. 生态学报, 2015, 35(14): 4570-4578.]

[ 5 ] Van Apeldoorn ME, Van Egmond HP, Speijers GJ et al. Toxins of Cyanobacteria. Molecular Nutrition \& Food Research, 2007, 51(1): 7-60.

[6] Gupta N, Pant SC, Vijayaraghavan R et al. Comparative toxicity evaluation of cyanobacterial cyclic peptide toxin microcystin variants ( LR, RR, YR) in mice. Toxicology, 2003, 188(2/3) : 285-296.

[ 7 ] Neilan BA, Pearson LA, Muenchhoff J et al. Environmental conditions that influence toxin biosynthesis in Cyanobacteria. Environmental Microbiology, 2013, 15(5): 1239-1253.

[ 8 ] Xue Q, Steinman AD, Su X et al. Temporal dynamics of microcystins in Limnodrilus Hoffmeisteri, a dominant Oligochaete of hypereutrophic Lake Taihu, China. Environmental Pollution, 2016, 213 : 585-593. 
[ 9 ] Rivasseau C, Martins S, Hennion MC. Determination of some physicochemical parameters of Microcystins (Cyanobacterial Toxins) and trace level analysis in environmental samples using liquid chromatography. Journal of Chromatography A, 1998, 799(1/2): 155-169.

[10] Tai ND, Lam PKS, Shaw GR et al. Toxicology and risk assessment of freshwater Cyanobacterial (Blue-Green Algal) toxins in water. Reviews of Environmental Contamination \& Toxicology, 2000, 163(163) : 113.

[11] Azevedo SM, Carmichael WW, Jochimsen EM et al. Human intoxication by Microcystins during renal dialysis treatment in Caruaru-Brazil. Toxicology, 2002, 181: 441-446.

[12] Carmichael WW, Azevedo S, An JS et al. Human fatalities from cyanobacteria: Chemical and biological evidence for cyanotoxins. Environmental Health Perspectives, 2001, 109(7): 663.

[13] Zhou XF, Dong ZH, Yu SZ. A study of influencing factors on high incidence of liver cancer in Taixing. China Cancer, 1999, 8(3) : 350-351. [周学富, 董志辉, 俞顺章. 泰兴地区肝癌高发因素研究. 中国肿瘤, 1999, 8(3): 350-351.]

[14] Lu WG, Lin WY. Investigation on primary liver cancer mortality trends and the high-risk factors in 1969-1999 in Haimen. Med J Commun, 2001, 15(5) : 469-470. [陆卫根, 林文尧. 海门市 1969 1999 年原发性肝癌死亡率趋势及高发因 素的探讨. 交通医学, 2001, 15(5): 469-470.]

[15] Zhang Y, Lin S, Qian X et al. Temporal and spatial variability of chlorophyll a concentration in Lake Taihu using modis time-series data. Hydrobiologia, 2011, 661(1) : 235-250.

[16] Qin B, Li W, Zhu G et al. Cyanobacterial bloom management through integrated monitoring and forecasting in large shallow eutrophic Lake Taihu (China). Journal of Hazardous Materials, 2015, 287: 356-363.

[17] Wang JJ, Yang J, Xian QM et al. Characteristic of microcystin distributions and its relationships with environmental factors in Lake Taihu. J Lake Sci, 2011, 23(4) : 513-519. DOI:10.18307/2011.0404. [王经结, 杨佳, 鲜啟鸣等. 太湖微囊 藻毒素时空分布特征及与环境因子的关系. 湖泊科学, 2011, 23(4) : 513-519.]

[18] Su X, Xue Q, Steinman A et al. Spatiotemporal dynamics of Microcystin variants and relationships with environmental parameters in Lake Taihu, China. Toxins, 2015, 7(8) : 3224

[19] Huang XC, Luo HD, Huang PZ et al. Current status of microcystin contamination in source water and finished water and its environmental factors in China. J Environ Hyg, 2014, 4(5): 494-498. [黄晓淳, 骆和东, 黄培枝等. 我国水源水及 饮用水中微囊藻毒素的污染现状及影响因素研究. 环境卫生学杂志, 2014, 4(5): 494-498.]

[20] Jin XC, Tu QY eds. Standards of eutrophication survey in lake. The second edition. Beijing: China Environmental Science Press, 1990. [ 金相灿, 屠清瑛. 湖泊富营养化调查规范: 第二版. 北京: 中国环境科学出版社, 1990.]

[21] Chen YW, Chen KM, Hu YH. Discussion on possible error for phytoplankton chlorophyll-a concentration analysis using hot-ethanol extraction method. J Lake Sci, 2006, 18(5) : 550-552. DOI: 10.18307/2006.0519. [陈宇炜, 陈开宁, 胡耀 辉. 浮游植物叶绿素 a 测定的“热乙醇法” 及其测定误差的探讨. 湖泊科学, 2006, 18(5) : 550-552.]

[22] Wang C, Peng T, Lü YB et al. Survey and preliminary health risk assessment of drinking water and water resources in a city of Jiangnan. Envion Chem, 2014, 33(7): 1237-1238. [王超, 彭涛, 吕怡兵等. 江南某城市饮用水及其水源水中 微囊藻毒素调查及初步健康风险评价. 环境化学, 2014, 33(7): 1237-1238.]

[23] You HH, Xiao B, Zhang YP et al. Assenment of human health risk about trihalomethanes in drinking water of a certain district in Foshan. Contemp Med , 2011, 17(23): 156-158. [尤汉虎, 肖兵, 张艳萍等. 佛山某区饮用水三卤甲烷健 康风险评价. 当代医学, 2011, 17(23): 156-158.]

[24] Kenefick S, Hrudey S, Peterson H et al. Toxin release from Microcystis aeruginosa after chemical treatment. Water Science and Technology, 1993, 27(3/4) : 433-440.

[25] Tang CJ. Microcystins and derivative volatile organic surfocompound in Gonghu Bay, Lake Taihu[Dissertation]. Shanghai : East China Normal University, 2010. [唐承佳. 太湖贡湖湾水源地微囊藻毒素和含硫衍生污染物研究 [ 学位论文]. 上海: 华东师范大学, 2010.]

[26] Gao ZM, Zhao ZH, Zhang B et al. Seasonal variation of microcystins concentration and influencing factors in Meiliang Bay, Lake Taihu. Ecol Environ Sci, 2011, 20(6/7): 1063-1067. [高振美, 赵中华, 张波等. 太湖梅梁湾水体微囊藻 毒素含量的季节变化特征及其影响因素研究. 生态环境学报, 2011, 20(6/7) : 1063-1067.]

[27] Mao JY. Otherness of distribution characteristics and main impact factors of microcystins in typical eutrophication lakes [Dissertation]. Chengdu: Southwest Jiaotong University, 2012. [毛敬英. 典型富营养化湖泊微囊藻毒素分布特征及 
主要影响因子差异性分析 [学位论文]. 成都: 西南交通大学, 2012.]

[28] Xiao XF, Li WQ, Liu N et al. The harm and control of the cyanobacteria toxin in eutrophication water. J China Inst Water Resour Hydropower Res, 2005, 3(2) : 116-123. [肖兴富, 李文奇, 刘娜等. 富营养化水体中蓝藻毒素的危害及其控 制. 中国水利水电科学研究院学报, 2005, 3(2) : 116-123.]

[29] Liu C. Study on the content of microcystins in the water source of Shanghai and the optimal removal mathods[Dissertation]. Shanghai: Tongji University, 2007. [刘成. 微囊藻毒素在上海市水源地的分布状况及去除研究 [学位论文]. 上海: 同济大学, 2007.]

[30] Xu HP, Yang GJ, Zhou J et al. Effect of nitrogen and phosphorus concentration on colony growth of Microcystis flos-aquae in Lake Taihu. J Lake Sci, 2014, 26(2) : 213-220. DOI:10.18307/2014.0207. [许慧萍, 杨桂军, 周健等. 氮、磷浓度 对太湖水华微囊藻 (Microcystis flos-aquae) 群体生长的影响. 湖泊科学, 2014, 26(2) : 213-220.]

[31] Rinta-Kanto JM, Konopko EA, DeBruyn JM et al. Lake Erie Microcystis: Relationship between Microcystin production, dynamics of genotypes and environmental parameters in a large lake. Harmful Algae, 2009, 8(5) : 665-673.

[32] Lee TA, Rollwagen-Bollens G, Bollens SM et al. Environmental influence on cyanobacteria abundance and microcystin toxin production in a shallow temperate lake. Ecotoxicology and Environmental Safety, 2015, 114: 318-325.

[33] Sivonen K. Effects of light, temperature, nitrate, orthophosphate, and bacteria on growth of and hepatotoxin production by Oscillatoria agardhii strains. Applied and Environmental Microbiology, 1990, 56(9) : 2658-2666.

[34] Utkilen H, Gjølme N. Iron-stimulated toxin production in Microcystis aeruginosa. Applied and Environmental Microbiology, 1995, 61(2): 797-800.

[35] Yu SZ, Zhao N, Zi XL et al. The relationship cyanotoxin (Microcystin, MC) in pond-ditch water and primary liver cancer in China. Chin J Oncol, 2001, 23(2) : 96-99. [俞顺章, 赵宁, 资晓林等. 饮水中微囊藻毒素与我国原发性肝癌关 系的研究. 中华肿瘤杂志, 2001, 23(2): 96-99.]

[36] Liao J, Nong QQ, Hu XM et al. Serum microcystin-LR level and influencing factors in local residents of a high risk area of hepatocellular carcinoma in Guangxi. J Environ Health, 2016, 33(11): 1003-1006. [廖娟, 农清清, 胡新梅等. 广西肝 癌高发区人群血清微囊藻毒素-LR 水平及影响因素分析. 环境与健康杂志, 2016, 33(11) : 1003-1006.] 